\title{
Alistair Munro: Bounded Rationality and Public Policy: A Perspective from Behavioural Economics. Ian J. Bateman (ed.): The Economics of Non-Market Goods and Resources
}

\author{
Vol. 12. Springer, Dordrecht, Heidelberg, London and New York, \\ 2009, 319 pp. ISBN: 9781402094729
}

\author{
W. J. W. Botzen \\ Published online: 6 January 2011 \\ (C) The Author(s) 2011. This article is published with open access at Springerlink.com
}

The literature on behavioural economics has grown considerably over the last two decades. It has produced a non-negligible body of evidence that individual behaviour in many economic decisions in both hypothetical and actual markets shows regular deviations from the rational and selfish homo economicus. This has profound implications for mainstream economic models, such as expected utility theory, which assume rational behaviour, and which are being explored in this relatively young discipline (Starmer 2000). Alistair Munro's book on bounded rationality and public policy provides an important contribution to this emerging literature. The author has a broad scope and examines several implications of bounded rationality that are of interest for public economics. Related chapters of this book deal with, amongst other things, the optimal boundaries of the state, market regulation, merit wants, principle agent problems, fiscal policy, and pension and savings policies. In addition, several implications of bounded rationality that are relevant for environmental economists are addressed, mainly for non-market valuation research.

Paretian welfare economics is taken as a starting point in the introductory chapter, which relaxes one of its underlying principles of complete and consistent preferences of individuals that are in accordance with individual welfare. This principle is often violated for individuals who can be described as 'boundedly rational'. Munro follows many other economists by defining bounded rationality as 'behaviour that is not wholly explicable in terms of the satisfaction of complete and consistent preferences'. The implications of such behaviour for public economics are examined by posing the following fundamental questions: "How is it possible to make judgments about individual welfare when the information we have about preferences is often inconsistent?" "How can we predict individual and aggregate behaviour when standard models of the consumer are inaccurate?" "To what extent are policy-makers best modelled as boundedly rational actors?". Initial answers to these questions are provided by mainly drawing upon two strands of literature: that on experiments of individual decision making and that on valuation. Munro achieves a good balance between the empirical evidence

W. J. W. Botzen $(\varangle)$

Department of Environmental Economics, Institute for Environmental Studies, VU University,

Amsterdam, The Netherlands

e-mail: wouter.botzen@ivm.vu.nl 
on boundedly rational behaviour and the theoretical implications of the observed deviations from consumer rationality. Although the mathematics used is generally well explained, the reader may become confused sometimes by small mistakes in notation. The material is accessible for researchers or graduate students who want to be introduced to the topic, while several chapters tackle more advanced issues that can be of interest for an expert audience.

A broad range of evidence of behavior that contradicts the rational consumer model is outlined in the first chapters of the book and summarized in comprehensive tables. A central topic is the influence of frames on individual choice, which can be described as the 'framing effect'. This effect occurs if the way a decision problem is presented to subjects affects their choices in a manner that suggests that preferences differ between contexts, which are called 'frames'. Commonly, individuals show preference reversals where the relative valuation of items depends on the means by which the value is elicited. Such frame-dependent preferences are inconsistent with the standard Hicksian model of the consumer and would obviously complicate the task of environmental valuation. A related failure of the Hicksian model is reference-dependent preferences, which means that preferences are conditional on the reference point - typically the initial endowment of goods - from which individuals express valuation. An example of this behaviour which is extensively discussed by Munro is the common discrepancy in environmental valuation studies of the willingness-to-accept (WTA) compensation for giving up a good and the willingness-to-pay (WTP) for the same good, with the WTA being greater than the WTP. Behavioural models are introduced that relax properties of frame neutrality and reference independence, notably prospect theory developed by Nobel laureate Kahneman and his colleague Tversky, where preferences can differ if outcomes are framed as losses instead of gains from a specified reference point (Tversky and Kahneman 1991). Munro convincingly shows that the evidence of deviations from the rational consumer is widespread and applies to experiments with and without economic incentives, and to field experiments, as well as to real markets. The presented evidence supports the notion that, although repetitive choices, for example in markets, can reduce some of the deviations from rational behaviour, learning effects in repetitive settings are limited, and bounded rationality persists in many cases.

The evidence on bounded rationality can be interpreted in two different ways: the deviations from rationality may be caused by unreliable measurement or individuals really do not have complete and coherent preferences. Munro asserts that both interpretations are likely to have some truth in practice. The first interpretation obviously has implications for the way welfare measures should be elicited, for example, by means of economic experiments or stated and revealed preference studies. It suggests that researchers should aim to refine methods of welfare elicitation in order to prevent behavioural anomalies, such as framing effects.

The second interpretation that individual preferences are in fact incoherent can have profound implications for the equilibrium conditions of markets and welfare economics. Munro presents a theoretical analysis of how reference-dependent preferences can influence market outcomes in a partial and general equilibrium model. This formal exercise shows that price signals in markets may not be reliable signals of underlying welfare values, which would challenge the commonly viewed superiority of revealed preference methods used in environmental valuation. Traditional market failures, such as negative externalities, are also likely to apply to markets with boundedly rational individuals, while policy that corrects for market failures may be complicated if individuals are not fully rational and fail to maximize their returns. Nevertheless, an interesting contribution of Munro, and a centrepiece of this book, is that theorizing about welfare optimums in a general equilibrium framework is still possible with reference-dependent preferences. If choices or preferences provide some 
information about underlying welfare orderings, then a notion similar to the fundamental result of welfare economics follows, meaning that equilibrium of competitive markets can result in a Pareto welfare optimum.

A complex problem for a social planner is how to deal with information about individual preferences or welfare that depends on elicitation frames, and in the extreme cases, has a conflicting ordering of policy alternatives. Several solutions for this are put forward: switching to a theory of welfare that is not associated with preference satisfaction; allowing individual welfare to be frame-dependent; refraining from ordering preferences altogether; defining optimal or golden frames under which welfare is to be elicited; aggregating contradictory evidence; and using additional evidence on welfare to aggregate information. All of these approaches have their disadvantages, but the last two focusing on aggregation seem promising, especially if patterns of framing effects are simple and elicitation frames can incentivize choice and guide optimal learning of preferences. While Munro certainly provides an insightful discussion of these topics, he readily acknowledges that more research is needed "particularly on the issue of how to aggregate conflicting preference information".

I found the last chapter on the evaluation of methods to elicit preferences of boundedly rational individuals a refreshing contribution to environmental valuation research. Munro discusses the different techniques used in contingent valuation (CV) studies to elicit preferences: for instance, dichotomous choice, bidding games, and open-ended valuation questions, along with their associated anomalies. From this evaluation, it follows that setting a single golden elicitation frame is complicated, since each frame has its methodological strengths and weaknesses. As a result, part of the variance in welfare estimates in CV is the result of frame variation caused by the difference between actual preferences and preferences elicited by means of a particular frame used by the researcher. Considering this frame variation, Munro introduces a method to estimate the superiority of CV, citizen's jury, or partial valuation based on revealed preference data. Useful numerical examples are provided to illustrate this procedure of selecting the most sound research method in the light of the evidence on frame variation.

The main contribution of the book is to provide the reader with initial insights into the implications of bounded rationality for public policy. While touching upon many different fields, it does not provide final answers to several of the important questions raised, which results in a complex picture with several unresolved issues. This is, in principle, not a shortcoming of this book and is partly due to the current standing of this discipline. The contribution of this book is important to the emerging literature on bounded rationality in economic models, and it provides a useful platform for future research on this topic. Relevant topics for this are about aggregating frame-dependent preferences in valuation studies and the implications of bounded rationality for managing environmental risks. The latter is largely unexplored by Munro, and few other studies exist, while it is crucial, for example, in designing climate change mitigation and adaptation policies (Botzen and van den Bergh 2009). A major strength of this book is that it calls for a rethinking of assumptions of individual behaviour in economic theory, and that it provides a good foundation for economic theorizing in a world with boundedly rational people.

Open Access This article is distributed under the terms of the Creative Commons Attribution Noncommercial License which permits any noncommercial use, distribution, and reproduction in any medium, provided the original author(s) and source are credited. 


\section{References}

Botzen WJW, van den Bergh JCJM (2009) Bounded rationality, climate risks and insurance: is there a market for natural disasters? Land Econ 85(2):266-279

Starmer C (2000) Developments in non-expected utility theory: the hunt for a descriptive theory of choice under risk. J Econ Lit 38(2):332-382

Tversky A, Kahneman D (1991) Loss aversion in riskless choice: a reference-dependent model. Quart J Econ 106(4):1039-1061 